\title{
The GASP-WEBT monitoring of 3C 454.3 during the 2008 optical-to-radio and $\gamma$-ray outburst ${ }^{\star}$
}

\author{
M. Villata ${ }^{1}$, C. M. Raiteri ${ }^{1}$, M. A. Gurwell ${ }^{2}$, V. M. Larionov ${ }^{3,4,5}$, O. M. Kurtanidze ${ }^{6}$, M. F. Aller ${ }^{7}$, A. Lähteenmäki ${ }^{8}$, \\ W. P. Chen ${ }^{9}$, K. Nilsson ${ }^{10}$, I. Agudo ${ }^{11}$, H. D. Aller ${ }^{7}$, A. A. Arkharov ${ }^{4}$, U. Bach ${ }^{12}$, R. Bachev ${ }^{13}$, P. Beltrame ${ }^{14}$, \\ E. Benítez ${ }^{15}$, C. S. Buemi ${ }^{16}$, M. Böttcher ${ }^{17}$, P. Calcidese ${ }^{18}$, D. Capezzali19 ${ }^{19}$, D. Carosati1 ${ }^{19}$, D. Da Rio ${ }^{14}$, A. Di Paola ${ }^{20}$, \\ M. Dolci ${ }^{21}$, D. Dultzin ${ }^{15}$, E. Forné22, J. L. Gómez ${ }^{11}$, V. A. Hagen-Thorn ${ }^{3,5}$, A. Halkola ${ }^{10}$, J. Heidt ${ }^{23}$, D. Hiriart ${ }^{15}$, \\ T. Hovatta ${ }^{8}$, H.-Y. Hsiao ${ }^{9}$, S. G. Jorstad ${ }^{24}$, G. N. Kimeridze ${ }^{6}$, T. S. Konstantinova ${ }^{3}$, E. N. Kopatskaya ${ }^{3}$, E. Koptelova ${ }^{9}$, \\ P. Leto ${ }^{16}$, R. Ligustri ${ }^{14}$, E. Lindfors ${ }^{10}$, J. M. Lopez ${ }^{15}$, A. P. Marscher ${ }^{24}$, M. Mommert ${ }^{23}$, R. Mujica ${ }^{25}$, \\ M. G. Nikolashvili ${ }^{6}$, N. Palma ${ }^{17}$, M. Pasanen ${ }^{10}$, M. Roca-Sogorb ${ }^{11}$, J. A. Ros ${ }^{22}$, P. Roustazadeh ${ }^{17}$, A. C. Sadun ${ }^{26}$,

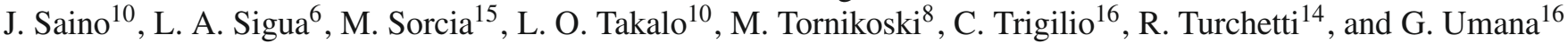

(Affiliations can be found after the references)

Received 19 June 2009 / Accepted 12 August 2009

\section{ABSTRACT}

\begin{abstract}
Context. Since 2001, the radio quasar 3C 454.3 has undergone a period of high optical activity, culminating in the brightest optical state ever observed, during the 2004-2005 outburst. The Whole Earth Blazar Telescope (WEBT) consortium has carried out several multifrequency campaigns to follow the source behaviour.

Aims. The GLAST-AGILE Support Program (GASP) was born from the WEBT to provide long-term continuous optical-to-radio monitoring of a sample of $\gamma$-loud blazars, during the operation of the AGILE and GLAST (now known as Fermi GST) $\gamma$-ray satellites. The main aim is to shed light on the mechanisms producing the high-energy radiation, through correlation analysis with the low-energy emission. Thus, since 2008 the monitoring task on 3C 454.3 passed from the WEBT to the GASP, while both AGILE and Fermi detected strong $\gamma$-ray emission from the source. Methods. We present the main results obtained by the GASP at optical, mm, and radio frequencies in the 2008-2009 season, and compare them with the WEBT results from previous years.

Results. An optical outburst was observed to peak in mid July 2008, when Fermi detected the brightest $\gamma$-ray levels. A contemporaneous mm outburst maintained its brightness for a longer time, until the $\mathrm{cm}$ emission also reached the maximum levels. The behaviour compared in the three bands suggests that the variable relative brightness of the different-frequency outbursts may be due to the changing orientation of a curved inhomogeneous jet. The optical light curve is very well sampled during the entire season, which is also well covered by the various AGILE and Fermi observing periods. The relevant cross-correlation studies will be very important in constraining high-energy emission models.
\end{abstract}

Key words. galaxies: active - galaxies: quasars: general - galaxies: quasars: individual: 3C 454.3 - galaxies: jets

\section{Introduction}

Blazars (i.e. flat-spectrum radio quasars and BL Lacertae objects) constitute a specific class of radio-loud active galactic nuclei whose highly variable emission is dominated by relativistically-beamed non-thermal radiation from a plasma jet. Blazars are detected at all wavelengths, from the radio to the $\gamma$-ray band. The low-energy non-thermal emission (from radio to optical, or sometimes to UV/X-rays) is due to synchrotron radiation, while the higher-energy emission is usually ascribed to inverse-Compton scattering of soft photons by the synchrotron-emitting relativistic electrons in the jet. According to synchrotron-self-Compton (SSC) models, the soft photons are the synchrotron photons themselves, while in external-Compton (EC) models, seed photons come from outside the jet, in particular from the accretion disc or the broad line region. The different predictions on the multifrequency behaviour of the source

* The radio-to-optical data presented in this paper are stored in the GASP-WEBT archive; for questions regarding their availability, please contact the WEBT President Massimo Villata (villata@oato.inaf.it). from different models can be tested by the results of coordinated multiwavelength campaigns, coupling the high-energy data from satellite observations with ground-based radio-tooptical data. In 1997, during the operation of the Compton Gamma Ray Observatory (CGRO, 1991-2000) satellite, this was one of the main motivations that led to the birth of the Whole Earth Blazar Telescope (WEBT) ${ }^{1}$, which involves a large number of telescopes at different longitudes, to obtain continuous monitoring during campaigns dedicated to single sources. Ten years later, in 2007, following the launch of the $\gamma$-ray satellite Astro-rivelatore Gamma a Immagini LEggero (AGILE), and in view of the anticipated launch of the Gamma-ray Large Area Space Telescope (GLAST, then renamed as the Fermi Gamma-ray Space Telescope), the WEBT started a new project: the GLAST-AGILE Support Program (GASP; see e.g. Villata et al. 2008b). Its primary aim is to provide long-term continuous optical-to-radio monitoring of a list of $28 \gamma$-loud blazars, during the operation of these two satellites, by means of selected WEBT telescopes.

\footnotetext{
${ }^{1}$ http://www.oato.inaf.it/blazars/webt/
} 


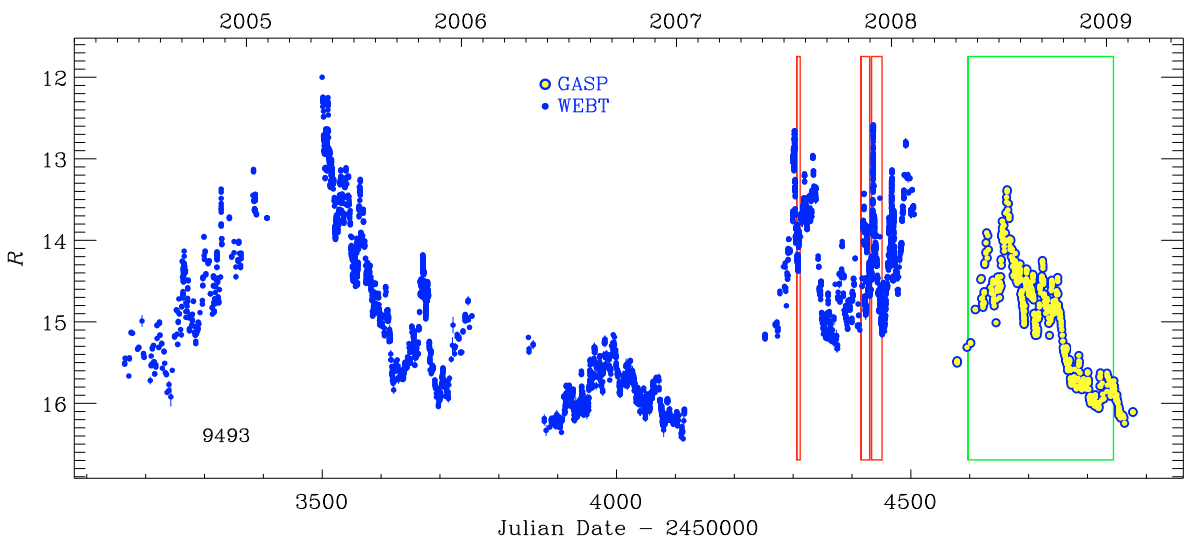

Fig. 1. $R$-band light curve of $3 \mathrm{C} 454.3$ in the last five observing seasons, composed with data from the WEBT campaigns (blue dots) and from the GASP monitoring (yellow-filled circles); the boxes indicate various periods of $\gamma$-ray detection, as described in the text.
The flat-spectrum radio quasar $3 \mathrm{C} 454.3(z=0.859)$ is one of the most studied blazars, especially after the observation of an exceptional bright state from the $\mathrm{mm}$ band to X-rays in May 2005. That unprecedented outburst was monitored from the radio to the optical band by the WEBT, whose results were published by Villata et al. (2006) together with Chandra observations and VLBA imaging ${ }^{2}$. The WEBT continued to monitor the subsequent high radio activity; an interpretation of the opticalradio correlation was proposed by Villata et al. (2007). The subsequent observing seasons showed an alternation of quiescent and active states; the relevant WEBT monitoring and spectral energy distribution (SED) results were presented and analysed in Raiteri et al. (2007, 2008a,b) together with optical-UV and $\mathrm{X}$-ray observations by the XMM-Newton and Swift satellites and spectroscopic monitoring in the near-IR. During the renewed optical activity of the second half of 2007, AGILE detected the source several times, at the brightest levels ever observed up to that time, as shown by Vercellone et al. $(2008,2009)$ and Donnarumma et al. (2009). In the last two papers, the authors also performed a cross-correlation analysis between the $\gamma$-ray fluxes in November and December 2007 and the corresponding WEBT optical data, showing a possible delay of the $\gamma$-ray emission of about 1 day or less. In particular, the detection of an exceptionally fast and strong flare in both the optical and $\gamma$-ray bands on December 12 seems to constrain the time lag to be less than $12 \mathrm{~h}$.

Thus, the WEBT followed the radio-to-optical behaviour of 3C 454.3 until February 2008; since then, the GASP continued this task in the last observing season 2008-2009, as part of its 28-source monitoring effort. Between late May and late June 2008, Donnarumma et al. (2008), Vittorini et al. (2008), and Gasparrini et al. (2008) reported on various episodes of $\gamma$-ray activity detected by AGILE. In July, the GASP observed a bright optical-NIR flare accompanied by $\mathrm{mm}$ and $\mathrm{cm}$ radio activity (Villata et al. 2008a). In the meantime, GLAST/Fermi-GST started its operation and the Large Area Telescope (LAT), turned on in late June, promptly detected high $\gamma$-ray activity, at unprecedented emission levels, especially during the optical flare (Tosti et al. 2008). Pittori et al. (2008) reported on the further observation by AGILE in late July. Following the optical-to-radio activity detected in July, the GASP continued to observe an increasing flux at high radio frequencies in the subsequent months, leading to the highest levels ever recorded at $43 \mathrm{GHz}$ (Bach et al. 2008).

Abdo et al. (2009) present the results of the first three months of Fermi-LAT observations of 3C 454.3, when the

\footnotetext{
${ }^{2}$ Observations by the INTEGRAL and Swift satellites and by the REM telescope were presented in Pian et al. (2006), Giommi et al. (2006), and Fuhrmann et al. (2006), respectively.
}

source showed unprecedentedly strong and highly-variable $\gamma$-ray emission, with a peak flux of $F_{E>100 \mathrm{MeV}} \approx 1.2 \times$ $10^{-5}$ photons $\mathrm{cm}^{-2} \mathrm{~s}^{-1}$. Their light curve covers (with a quasidaily sampling) the period July $1-$ October 5 , and the similarities with the optical light curve presented in this letter are evident. However, this letter is focused on the multifrequency behaviour observed by the GASP in the 2008-2009 observing season, and any consideration and analysis on the optical- $\gamma$ correlation and its impact on theoretical models is deferred to subsequent papers in collaboration with the AGILE and Fermi teams. In Sect. 2 we present the optical-to-radio observations with a selection of light curves; in Sect. 3 we discuss the results.

\section{Optical-to-radio observations and results}

We calibrated the optical $R$-band magnitudes with respect to Stars 1-4 from the photometric sequence by Raiteri et al. (1998). Figure 1 shows the $R$-band data collected by the GASP in the 2008-2009 observing season (from April 2008 to February 2009; yellow-filled circles), together with WEBT data during the previous four seasons, since the big 2004-2005 outburst (blue dots; data from Villata et al. 2006, 2007; Raiteri et al. 2007, 2008a,b). In total, we assembled about 9500 data points. The participating GASP optical observatories in the 2008-2009 season were: Abastumani, Lulin, Armenzano, Crimean, Roque de los Muchachos (KVA), Belogradchik, San Pedro Martir, St. Petersburg, Kitt Peak (MDM), Teide (BRT), Sabadell, Talmassons, Calar Alto ${ }^{3}$, L'Ampolla, New Mexico Skies, Valle d'Aosta, and Tuorla. Near-IR $J H K$ data were taken at Campo Imperatore. In the figure, the boxes indicate periods of $\gamma$-ray detection: the red ones are those by AGILE in 2007 (Vercellone et al. 2008, 2009; Donnarumma et al. 2009), while the green one represents the total period covered by AGILE and/or Fermi in 2008 - early 2009.

The GASP light curve from April 2008 to February 2009 is better displayed in Fig. 2, where also the details of the $\gamma$-ray observing periods are given: red boxes are the AGILE ones, whereas the blue box indicates the July 1-October 5 period covered by the light curve in Abdo et al. (2009) ${ }^{4}$. The optical flux started to increase in mid May and a first, noticeable flare peaked in mid June, at the end of the first AGILE period. A further, brighter flare was observed to double-peak on July 7-10

\footnotetext{
${ }^{3}$ Calar Alto data were acquired as part of the MAPCAT (Monitoring AGN with Polarimetry at the Calar Alto Telescopes) project.

4 The Fermi-LAT monitoring has continued beyond this period; preliminary light curves are shown at http://fermi.gsfc.nasa.gov/ ssc/data/access/lat/msl_lc/.
} 


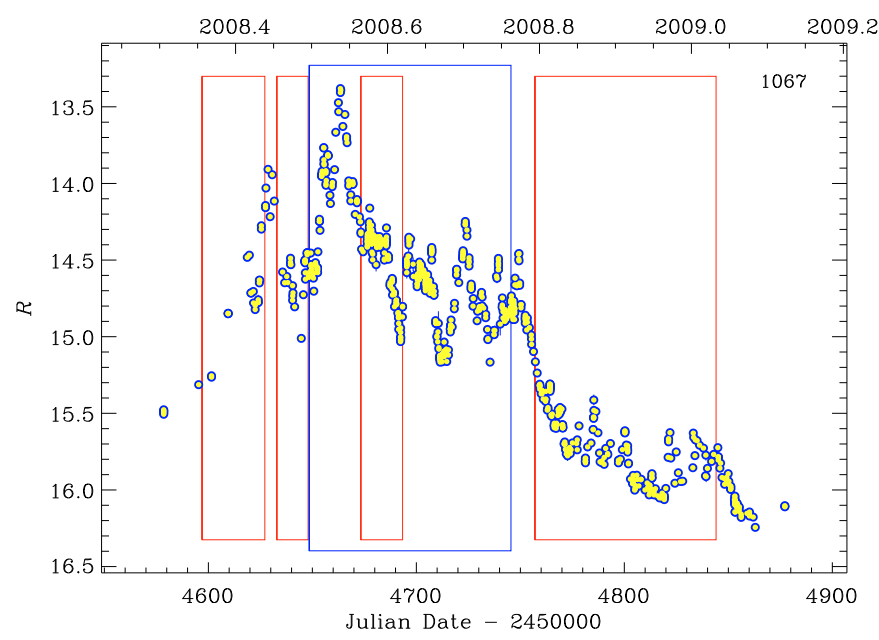

Fig. 2. Enlargement of the last season of the $R$-band light curve shown in Fig. 1, during the GASP monitoring; see text for explanation of the red and blue boxes.

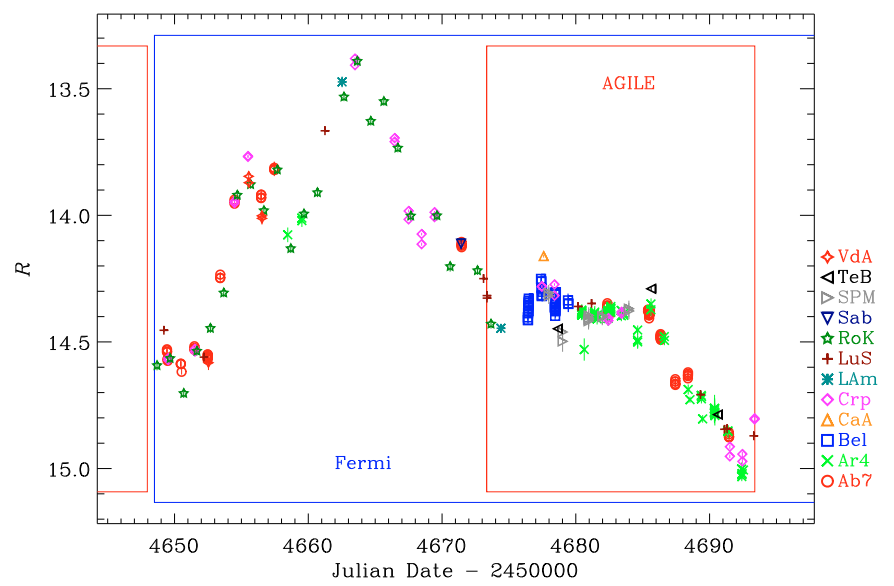

Fig. 3. Further enlargement of the $R$-band light curve around the brightest phase of the 2008 outburst, well monitored also by Fermi and AGILE; different symbols indicate different observatories.

(JD 2454 655-57), followed by the brightest phase of the outburst, peaking around July 16.1 (JD 2454 663.6). This brightest part of the 2008 outburst is visible in more detail in Fig. 3, which reports the period from the start of the Abdo et al. (2009) $\gamma$-ray light curve (July 1.0, JD $=2454648.5$ ) until the end of the third 2008 AGILE period (August 14.88, JD = 2454693.38 ), with symbols differentiating the various observatories. Then the optical flux started to drop with a decreasing trend with several flares superposed, until the end of the season.

The GASP monitored the source also at $\mathrm{mm}$ and $\mathrm{cm}$ wavelengths: 345 and $230 \mathrm{GHz}\left(\mathrm{SMA}^{5}\right), 43 \mathrm{GHz}$ (Noto), $37 \mathrm{GHz}$ (Metsähovi), $22 \mathrm{GHz}$ (Medicina), $14.5 \mathrm{GHz}$ (UMRAO), 8 and $5 \mathrm{GHz}$ (UMRAO and Medicina). In Fig. 4, we show the bestsampled and most-significant light curves from late March 2008 to February 2009, at 230, 37, and $14.5 \mathrm{GHz}^{6}$. The optical outburst was accompanied by very high activity at these lower frequencies; in particular, the $37 \mathrm{GHz}$ data show levels never observed before, brighter than the previous historical maximum of early 2006. The $\mathrm{mm}-\mathrm{cm}$ outbursts appear to be delayed with

\footnotetext{
5 These data were obtained as part of the normal monitoring program initiated by the SMA (see Gurwell et al. 2007).

6 The other, less-sampled light curves show similar/intermediate trends.
}

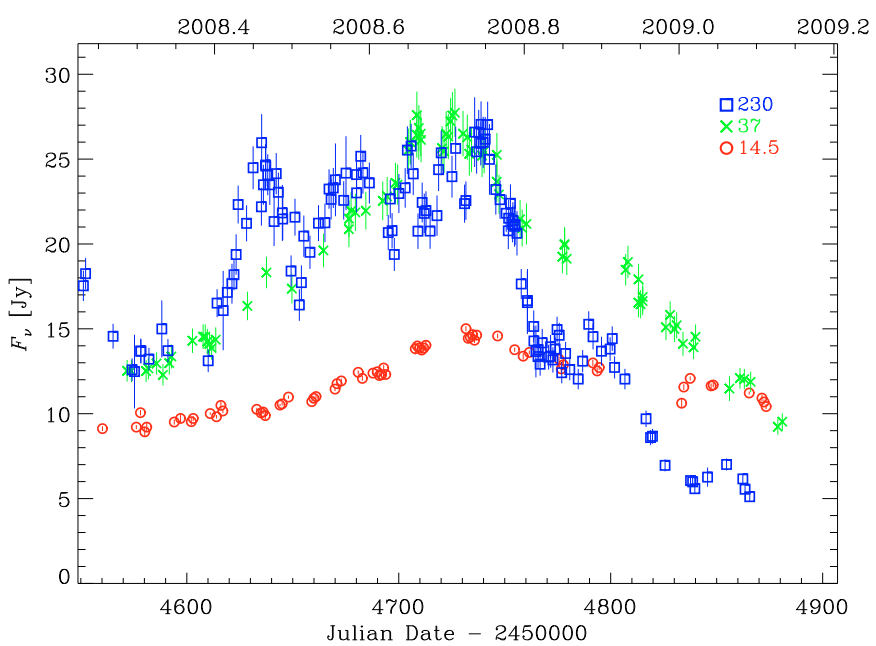

Fig. 4. GASP light curves of 3C 454.3 from late March 2008 to February 2009 at $230 \mathrm{GHz}$ (blue squares), $37 \mathrm{GHz}$ (green crosses), and $14.5 \mathrm{GHz}$ (red circles).
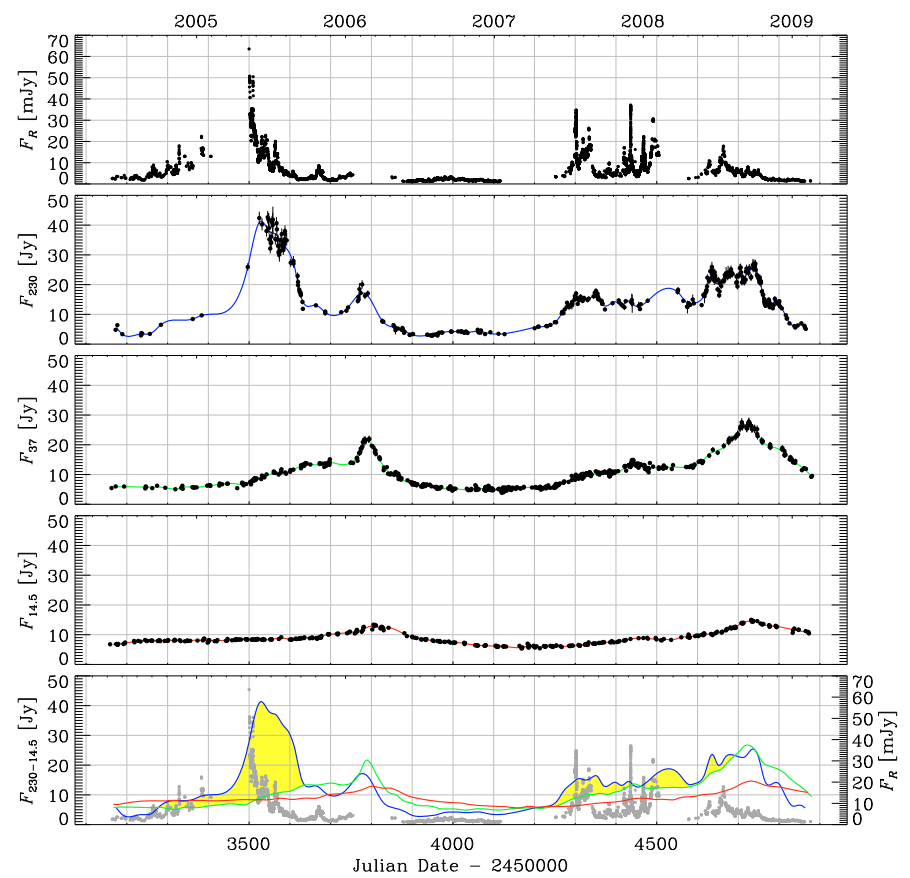

Fig. 5. $R$-band flux-density (de-reddened) light curve (top, mJy) and $\mathrm{mm}-\mathrm{cm}$ light curves (Jy) at 230, 37, and $14.5 \mathrm{GHz}$ from June 2004 to February 2009; in the bottom panel the optical data are reported together with the cubic spline interpolations through the 15-day binned data at 230 (blue line), 37 (green line), and 14.5 (red line) GHz; the regions where the $230 \mathrm{GHz}$ spline exceeds the $37 \mathrm{GHz}$ one are highlighted in yellow.

respect to the optical outburst, especially in the decreasing phase, with the delay increasing with wavelength.

Figure 5 displays the flux behaviour at optical, mm, and radio frequencies in the last five seasons, starting from the 2004-2005 historical outburst, including the WEBT data from Villata et al. (2006, 2007) and Raiteri et al. (2007, 2008a,b). Compared to the previous optical activity, the 2008 outburst appears relatively modest, while the corresponding $\mathrm{mm}(230 \mathrm{GHz})$ outburst is much more spectacular, and the radio levels at 37 and $14.5 \mathrm{GHz}$ are higher than in the previous events. 


\section{Discussion}

The unprecedented optical outburst of 2005 was followed by an equally exceptional $\mathrm{mm}$ event, but, at lower frequencies, the signature of this event was only a moderate flux increase. According to Villata et al. (2007), the different appearance of the outburst (which is caused by an emitting zone moving along the jet) at different frequencies was due to different viewing angles of the various emitting regions in the (curved) jet: smaller viewing angles yield a stronger Doppler enhancement of the flux. After that, an orientation change of the curved jet provided the extraordinary outburst at high radio frequencies of early 2006, as the counterpart of non-enhanced optical activity. This interpretation predicted a mild mm event, as one can now see in Fig. 5 (see also Raiteri et al. 2008b). In other words, the ratios between the fluxes at different frequencies depend on the curved jet orientation. Following this picture, the strong (but not exceptional) optical activity in the 2007-2008 season, accompanied by mild $\mathrm{mm}$ and $\mathrm{cm}$ activity, would represent a geometrical configuration slightly favouring high frequencies. Then (2008-2009), the initial optical brightness is soon suppressed in favour of the $\mathrm{mm}$ one, which continues to be bright for a longer period, until the radio frequencies also brighten, more than previously, due to a particularly small viewing angle. It seems that the jet curvature can allow only two bands (e.g. optical and $\mathrm{mm}$, or $\mathrm{mm}$ and $\mathrm{cm}$ ) to be contemporaneously strongly Doppler enhanced: if one of the outer bands (i.e. optical and radio) is enhanced, the other is non-enhanced (see also the model for BL Lacertae in Villata et al. 2009 and Fig. 4 therein). To make this evident, in the bottom panel of Fig. 5 we plotted the optical data and cubic spline interpolations through the $\mathrm{mm}$ and $\mathrm{cm}$ light curves together. The yellow areas highlight the regions where the $230 \mathrm{GHz}$ spline exceeds that at $37 \mathrm{GHz}$, i.e. when the mm emitting region is better aligned with the line of sight than the $\mathrm{cm}$ one. As expected, the larger the $230 / 37 \mathrm{GHz}$ flux ratio, the higher the corresponding optical activity, and the optical appears always low when the $230 / 37 \mathrm{GHz}$ ratio is less than 1 . A deeper, quantitative analysis of this correlation is beyond the aim of this short letter.

We have reported on the optical-to-radio flux behaviour of 3C 454.3 in the 2008-2009 season, as observed by the GASP, and have compared these results with the WEBT light curves of recent years, finding consistency with the curved jet scenario depicted by Villata et al. (2007). In particular, we have assembled a detailed optical light curve during and around the various detection periods by AGILE and Fermi, which will be very useful for cross-correlation studies with the $\gamma$-ray emission.

Acknowledgements. We thank the referee, R. Hartman, for useful suggestions. The Torino team acknowledges financial support by the Italian Space Agency through contract ASI-INAF I/088/06/0 for the Study of High-Energy Astrophysics. The Submillimeter Array is a joint project between the Smithsonian Astrophysical Observatory and the Academia Sinica Institute of Astronomy and Astrophysics and is funded by the Smithsonian Institution and the Academia Sinica. The St. Petersburg team acknowledges support from Russian Foundation for Basic Researches, grant 09-02-00092. AZT-24 observations at Campo Imperatore are made within an agreement between Pulkovo, Rome and Teramo observatories. This research has made use of data from the University of Michigan Radio Astronomy Observatory, which is supported by the National Science Foundation and by funds from the University of Michigan. The Metsähovi team acknowledges the support from the Academy of Finland. The research has been supported by the Taiwan National Science Council grant No. 96-2811-M-008-033. This paper is partly based on observations carried out at the German-Spanish Calar Alto Observatory, which is jointly operated by the MPIA and the IAA-CSIC. Acquisition of the MAPCAT data is supported in part by the Spanish "Ministerio de Ciencia e Innovación" through grant AYA2007-67626-C03-03. This work is partly based on observations with the Medicina and Noto radio telescopes operated by INAF - Istituto di Radioastronomia.

\section{References}

Abdo, A. A., Ackermann, M., Ajello, M., et al. 2009, ApJ, 699, 817

Bach, U., Gurwell, M. A., Leto, P., et al. 2008, The Astronomer's Telegram, 1849,1

Donnarumma, I., D’Ammando, F., Pacciani, L., et al. 2008, The Astronomer's Telegram, 1545, 1

Donnarumma, I., Pucella, G., Vittorini, V., et al. 2009, ApJ, submitted Fuhrmann, L., Cucchiara, A., Marchili, N., et al. 2006, A\&A, 445, L1 Gasparrini, D., Pittori, C., Verrecchia, F., et al. 2008, The Astronomer's Telegram, 1592, 1

Giommi, P., Blustin, A. J., Capalbi, M., et al. 2006, A\&A, 456, 911

Gurwell, M. A., Peck, A. B., Hostler, S. R., Darrah, M. R., \& Katz, C. A. 2007, in From Z-Machines to ALMA: (Sub)Millimeter Spectroscopy of Galaxies, ed. A. J. Baker, J. Glenn, A. I. Harris, J. G. Mangum, \& M. S. Yun, ASP Conf. Ser., 375, 234

Pian, E., Foschini, L., Beckmann, V., et al. 2006, A\&A, 449, L21

Pittori, C., Verrecchia, F., Vercellone, S., et al. 2008, The Astronomer's Telegram, 1634, 1

Raiteri, C. M., Villata, M., Lanteri, L., Cavallone, M., \& Sobrito, G. 1998, A\&AS, 130, 495

Raiteri, C. M., Villata, M., Larionov, V. M., et al. 2007, A\&A, 473, 819 Raiteri, C. M., Villata, M., Chen, W. P., et al. 2008a, A\&A, 485, L17 Raiteri, C. M., Villata, M., Larionov, V. M., et al. 2008b, A\&A, 491, 755

Tosti, G., Chiang, J., Lott, B., et al. 2008, The Astronomer's Telegram, 1628, 1 Vercellone, S., Chen, A. W., Giuliani, A., et al. 2008, ApJ, 676, L13 Vercellone, S., Chen, A. W., Vittorini, V., et al. 2009, ApJ, 690, 1018 Villata, M., Raiteri, C. M., Balonek, T. J., et al. 2006, A\&A, 453, 817 Villata, M., Raiteri, C. M., Aller, M. F., et al. 2007, A\&A, 464, L5

Villata, M., Raiteri, C. M., Larionov, V. M., et al. 2008a, The Astronomer's Telegram, 1625, 1

Villata, M., Raiteri, C. M., Larionov, V. M., et al. 2008b, A\&A, 481, L79

Villata, M., Raiteri, C. M., Larionov, V. M., et al. 2009, A\&A, 501, 455

Vittorini, V., Donnarumma, I., D'Ammando, F., et al. 2008, The Astronomer's Telegram, 1581, 1

1 INAF - Osservatorio Astronomico di Torino, Italy

2 Harvard-Smithsonian Center for Astrophysics, MA, USA

3 Astronomical Institute, St.-Petersburg State University, Russia

4 Pulkovo Observatory, Russia

5 Isaac Newton Institute of Chile, St.-Petersburg Branch, Russia

6 Abastumani Astrophysical Observatory, Georgia

7 Department of Astronomy, University of Michigan, MI, USA

8 Metsähovi Radio Observatory, Helsinki University of Technology

TKK, Finland

9 Institute of Astronomy, National Central University, Taiwan

10 Tuorla Observatory, Department of Physics and Astronomy, University of Turku, Finland

11 Instituto de Astrofísica de Andalucía, CSIC, Spain

12 Max-Planck-Institut für Radioastronomie, Germany

13 Institute of Astronomy, Bulgarian Academy of Sciences, Bulgaria

14 Circolo Astrofili Talmassons, Italy

15 Instituto de Astronomía, Universidad Nacional Autónoma de México, Mexico

16 INAF - Osservatorio Astrofisico di Catania, Italy

17 Astrophysical Institute, Department of Physics and Astronomy, Ohio University, OH, USA

18 Osservatorio Astronomico della Regione Autonoma Valle d'Aosta, Italy

19 Armenzano Astronomical Observatory, Italy

20 INAF - Osservatorio Astronomico di Roma, Italy

21 INAF - Osservatorio Astronomico di Collurania Teramo, Italy

22 Agrupació Astronòmica de Sabadell, Spain

23 ZAH, Landessternwarte Heidelberg-Königstuhl, Germany

24 Institute for Astrophysical Research, Boston University, MA, USA

25 INAOE, Mexico

26 Department of Physics, University of Colorado Denver, CO, USA 